\title{
Randomised study comparing extent of hypocarbia in preterm infants during conventional and patient triggered ventilation
}

\author{
K Luyt, D Wright, J H Baumer
}

\begin{abstract}
Aim-To determine whether patient triggered ventilation (PTV) leads to greater exposure to significant hypocarbia than conventional ventilation (CMV) in premature infants during the first 72 hours of life.

Methods-Infants of 32 weeks gestation or less were included. Randomisation yielded 74 infants on PTV and 68 infants on CMV. Arterial $\mathrm{PaCO}_{2}$ measurements were taken four hourly for the first 72 hours of life. Results-The mean $\mathrm{PaCO}_{2}$ levels on days 1 , 2 , and 3 were not significantly different between the two groups. The proportion of infants with $\mathrm{PaCO}_{2}$ levels of $3.33 \mathrm{kPa}$ or less did not differ between PTV and CMV infants. Mean percentages of infants with this level of hypocarbia at any time were $31.4 \%, 18.9 \%, 8.8 \%$ on days 1,2 , and 3 respectively. Cumulative hypocarbia, below a $3.33 \mathrm{kPa}$ threshold, was 0.0084 kPa.h (PTV) versus $0.0263 \mathrm{kPa.h}$ (CMV) per hour ventilated during the first 24 hours $(p=0.259)$. Risk factors associated with hypocarbia on day 1 were peak inspiratory pressure below $14 \mathrm{~cm} \mathrm{H}_{2} \mathrm{O}$ (odds ratio 4.79) as well as $\mathrm{FiO}_{2}$ below 0.30 (odds ratio 3.42).

Conclusion-Exposure to hypocarbia $\left(\mathrm{PaCO}_{2} 3.33 \mathrm{kPa}\right.$ or below) was not significantly different between PTV and CMV infants during the first 72 hours of life. Hypocarbia was common in both groups on day 1 and to a lesser extent on day 2 . Infants with the least requirements for ventilatory support were at highest risk of hypocarbia on day 1 of life. Preterm infants with mild hyaline membrane disease require a more aggressive approach to weaning on both modes of ventilation, followed by extubation to limit the risk of hypocarbia.
\end{abstract}

(Arch Dis Child Fetal Neonatal Ed 2001;84:F14-F17)

Keywords: patient triggered ventilation; intermittent positive pressure ventilation; hypocarbia

Carbon dioxide is an important regulator of cerebral blood flow in the preterm infant. ${ }^{1}$ Wyatt et al found a reduction in cerebral blood flow to be associated with lower $\mathrm{PaCO}_{2}$ levels and showed greater cerebrovascular sensitivity to hypocarbia with increasing gestational age. ${ }^{2}$

Several studies have shown an association between hypocarbia and neurodevelopmental sequelae $^{34}$ or periventricular leukomalacia ${ }^{5-8}$ in preterm infants.

Measures of significant hypocarbia have included one or more measurements of arterial $\mathrm{CO}_{2}\left(\mathrm{PaCO}_{2}\right)$ below $20 \mathrm{~mm} \mathrm{Hg}(2.67 \mathrm{kPa})^{5}$ or $25 \mathrm{~mm} \mathrm{Hg}(3.33 \mathrm{kPa}),{ }^{6}$ or a measure of the severity and duration of hypocarbia below a threshold of $25 \mathrm{~mm} \mathrm{Hg}(3.33 \mathrm{kPa})$, cumulative hypocarbia ( $\mathrm{mm} \mathrm{Hg}$.hours). ${ }^{7}$

Infants studied have been ventilated in a variety of modes, including intermittent mandatory ventilation ${ }^{3-68}$ and high frequency jet ventilation. $^{7}$

Two studies reported an association between lower $\mathrm{PaCO}_{2}$ levels and chronic lung disease of prematurity. One studied infants at 48 hours $^{9}$ and the other prior to surfactant administration. $^{10}$

Opinion in the literature is divided regarding the effect of trigger ventilation on arterial carbon dioxide tension. Two small studies ${ }^{11} 12$ conducted in the presurfactant and a third ${ }^{13}$ in the surfactant era showed significantly lower $\mathrm{PCO}_{2}$ levels in infants switched over from conventional to trigger ventilation. A similar study reported greater tidal volumes in premature infants with respiratory distress syndrome (RDS) on patient triggered ventilation (PTV), but no significant reduction in carbon dioxide concentrations. $^{14}$

The aim of this study was to establish whether PTV was associated with a greater risk of significant hypocarbia in premature infants. The trigger ventilation trial ${ }^{15}$ provided an opportunity to study this using routinely recorded arterial blood gas data.

\section{Patients and methods}

INFANTS

The study utilised all patients recruited by a single centre (Plymouth) within a multicentre trigger ventilation trial designed to compare PTV and conventional ventilation (CMV) in RDS. Infants less than 32 weeks gestation requiring ventilation within 72 hours of birth, with clinical and $x$ ray features compatible with respiratory distress syndrome (RDS), were eligible for trial entry. Infants with evidence of major congenital malformations and inhalational pneumonitis were excluded.

\section{RANDOMISATION}

The mode of ventilation was allocated by an independent nurse providing the telephone randomisation service, within six hours of commencing ventilation. Randomisation was performed in blocks of varying size. After randomisation four infants were changed from 
Table 1 Demographic data according to mode of ventilation

\begin{tabular}{|c|c|c|}
\hline & $P T V(n=74)$ & $C M V(n=68)$ \\
\hline Weight $(\mathrm{g})^{\star}$ & $1018(585-1935)$ & $1018(496-2235)$ \\
\hline Male & $39(53 \%)$ & $44(65 \%)$ \\
\hline Gestation $(w k)^{\star}$ & $28(23-31)$ & $28(23-31)$ \\
\hline Antenatal steroids (full course) & $48(65 \%)$ & $45(66 \%)$ \\
\hline Surfactant & $71(96 \%)$ & $68(100 \%)$ \\
\hline Synthetic surfactant & $62(87 \%)$ & $58(85 \%)$ \\
\hline CRIB score* & $7(1-18)$ & $7(1-18)$ \\
\hline
\end{tabular}

${ }^{\star}$ Median (range).

PTV to CMV: two because of failure to trigger, one because of failure to oxygenate, and one following a pulmonary haemorrhage. Analysis was performed on an intention to treat basis.

VENTILATION STRATEGY

The SLE 2000 jet ventilator was used in either CMV or PTV mode. Prior to randomisation infants were ventilated with the attending clinician's preferred mode and technique of ventilation. After randomisation the ventilator was switched to the allocated mode and ventilation was guided by the following protocol: peak inspiratory pressure was adjusted according to chest wall movement and blood gas results and positive end expiratory pressure set at $4-5 \mathrm{~cm}$ $\mathrm{H}_{2} \mathrm{O}$. In PTV mode an inspiratory time of $0.2-$ 0.3 seconds was chosen initially, the ventilator set to trigger each inspiratory effort with a back up rate of 35 breaths per minute. The back up rate could be increased in apnoeic infants. Those receiving CMV had ventilator rates set either to the infant's spontaneous respiratory rate, or at 80 breaths per minute, with instructions to alter the rate as required. Initial inspiratory times were set at 0.34 seconds. Weaning was achieved by reducing inspiratory pressures to a minimum of $8-12 \mathrm{~cm} \mathrm{H}_{2} \mathrm{O}$ on PTV and by reducing the inspiratory pressure to a minimum of $16 \mathrm{~cm} \mathrm{H}_{2} \mathrm{O}$ followed by reducing the ventilator rate on CMV.

The attending clinicians were guided by the ventilation protocol and used clinical judgement to manage suboptimal blood gas results. The magnitude of change in ventilator settings was left to the judgement of individual doctors. The use of either caffeine or theophylline and morphine was permissible. All infants were eligible for surfactant in accordance with the unit's protocol.

BLOOD GAS ANALYSIS

Blood gas values were measured from samples obtained from indwelling arterial catheters every four hours, and within one hour following changes in ventilator settings. Measurements taken prior to randomisation were excluded from the analysis. Transcutaneous $\mathrm{CO}_{2}$ electrodes were not routinely used in the study infants in the first few postnatal days.

DATA ANALYSIS

$\mathrm{PaCO}_{2}$ data analysis was performed retrospectively from intensive care charts. Cumulative hypocarbia for each infant was calculated using S-PLUS according to the method of Wiswell et $a l{ }^{7}$ Mean values were compared using Student's $t$ test for parametric data and the MannWhitney test for non-parametric data. When comparing differences in proportions the $\chi^{2}$ test was used. Analysis of variance was used to compare the difference in the pre- and post-randomisation $\mathrm{PaCO}_{2}$ measurements in four groups of infants: those switched from CMV to PTV and PTV to CMV as well as those remaining on either CMV or PTV. Logistic regression was used to evaluate the effect of several postnatal variables on the risk of developing hypocarbia on day 1 of life. Risk was expressed as an odds ratio with $95 \%$ confidence limits. Goodness of fit was determined by the Hosmer-Lemeshow statistic in which a high $\mathrm{p}$ value indicates an adequate fit of the data to the model.

All statistical analyses were conducted on SPSS. We considered $\mathrm{p}<0.05$ statistically significant.

ETHICAL CONSIDERATIONS

Written informed parental consent was sought prior to randomisation and local research ethics committee approval was obtained for the trigger ventilation trial. Blood gas estimations and all other management was delivered at the discretion of the clinicians within the research protocol outlined.

\section{Results}

Randomisation yielded 74 infants on PTV and 68 infants on CMV. Infants were comparable at trial entry (table 1). Similar respiratory rates and fractions of inspired oxygen $\left(\mathrm{FiO}_{2}\right)$ were measured, but median peak inspiratory pressures (PIP) were lower in the PTV group on days 1 and 2 respectively $\left(14.7 v 16.9 \mathrm{~cm} \mathrm{H}_{2} \mathrm{O}\right.$ $(\mathrm{p}=0.002)$ and $14.5 \quad v \quad 17.8 \quad \mathrm{~cm} \quad \mathrm{H}_{2} \mathrm{O}$ $(\mathrm{p}=0.008))$.

The proportion of infants with one or more episodes of hypocarbia $\left(\mathrm{PaCO}_{2} 3.33 \mathrm{kPa}\right.$ or below) was $34.7 \%$ (PTV) v $27.9 \%$ (CMV) on day $1,18.6 \%$ (PTV) $v 19.1 \%$ (CMV) on day 2 , and $9.1 \%(\mathrm{PTV})$ v $8.5 \%$ (CMV) on day 3 ( $p>0.05$ ). Overall $37.3 \%$ of all infants experienced inadvertent hypocarbia during the first 72 hours

The mean $\mathrm{PaCO}_{2}$ measured on day 1 was 4.8 $\mathrm{kPa}(\mathrm{PTV})$ v $5.19 \mathrm{kPa}(\mathrm{CMV})(\mathrm{p}=0.05)$ Mean $\mathrm{PaCO}_{2}$ measurements on days 2 and 3 were $5.89 \mathrm{kPa}(\mathrm{PTV}) v 5.85 \mathrm{kPa}(\mathrm{CMV})$ and $6.1 \mathrm{kPa}(\mathrm{PTV}) \approx 6.0 \mathrm{kPa}(\mathrm{CMV})$ respectively $(\mathrm{p}>0.05)$

The median area below the $3.33 \mathrm{kPa}$ threshold and above the $\mathrm{CO}_{2}$ curve (cumulative hypocarbia), was $0.0084 \mathrm{kPa} . \mathrm{h}(\mathrm{PTV}) v 0.0263$ $\mathrm{kPa} . \mathrm{h}$ (CMV) per hour ventilated during the first 24 hours $(p=0.259)$.

Analysis of variance showed no significant difference in mean pre- and postrandomisation $\mathrm{PaCO}_{2}$ levels in four groups of infants: those switched over from CMV to PTV or from PTV to CMV as well as those remaining on their prerandomisation mode of ventilation, either CMV or PTV.

Using univariate analyses we evaluated several factors considered to play a potential role in the development of hypocarbia $\left(\mathrm{PaCO}_{2}\right.$ $3.33 \mathrm{kPa}$ or below) on day 1 of life, including: mode of ventilation, gestation, weight, sex, antenatal steroids, use of surfactant, natural 
Table 2 Factors associated with hypocarbia

\begin{tabular}{|c|c|c|}
\hline Variable & Odds ratio & $\begin{array}{l}\text { (95\% confidence } \\
\text { interval) }\end{array}$ \\
\hline $\mathrm{PIP}<14 \mathrm{~cm} \mathrm{H}_{2} \mathrm{O}$ & 4.79 & $(1.87,12.31)$ \\
\hline PIP $14-16 \mathrm{~cm} \mathrm{H}_{2} \mathrm{O}$ & 2.33 & $(0.95,5.73)$ \\
\hline $\mathrm{PIP}>16 \mathrm{~cm} \mathrm{H}_{2} \mathrm{O}$ & Reference & \\
\hline $\mathrm{FiO}_{2}<0.30$ & 3.42 & $(1.41,8.34)$ \\
\hline $\mathrm{FiO}_{2} 0.30-0.60$ & 1.05 & $(0.33,3.25)$ \\
\hline $\mathrm{FiO}_{2}>0.60$ & Reference & \\
\hline
\end{tabular}

versus synthetic surfactant, peak inspiratory pressures, $\mathrm{FiO}_{2}$, respiratory rate, and illness severity (CRIB score ${ }^{16}$ ). We used stepwise logistic regression to assess whether any of these factors were independent predictors of hypocarbia.

The only factors incurring significant risk were mean peak inspiratory pressures and mean $\mathrm{FiO}_{2}$ on day 1. Both these factors had an inverse dose-response relationship with lower peak inspiratory pressures and $\mathrm{FiO}_{2}$ being associated with a higher risk of hypocarbia. The association of PIP and $\mathrm{FiO}_{2}$ with hypocarbia was not confounded by the other factors investigated. A multicolinear relation existed between $\mathrm{FiO}_{2}$ and PIP, and therefore these variables were entered separately in the final model.

Infants ventilated with mean peak inspiratory pressures less than $14 \mathrm{~cm} \mathrm{H}_{2} \mathrm{O}$ were 4.79 times as likely to develop hypocarbia as those with peak inspiratory pressures greater than 16 $\mathrm{cm} \mathrm{H}_{2} \mathrm{O}$. An $\mathrm{FiO}_{2}$ of less than 0.3 incurred 3.4 times the risk of an $\mathrm{FiO}_{2}$ of greater than 0.6 (table 2).

\section{Discussion}

The mean arterial carbon dioxide tension during the first 72 hours was not significantly different between the two groups; a nonsignificant trend towards lower mean $\mathrm{PaCO}_{2}$ levels was noted during PTV on day 1 $(p=0.05)$. No significant difference in cumulative hypocarbia was detected. The proportion of infants with $\mathrm{PaCO}_{2}$ levels of $3.33 \mathrm{kPa}$ or below did not differ between PTV and CMV infants. Hypocarbia was a regular occurrence on day 1 during both modes of ventilation with $31.3 \%$ of all infants experiencing $\mathrm{PaCO}_{2}$ levels of $3.33 \mathrm{kPa}$ or less. The risk factors associated with hypocarbia on day 1 were peak inspiratory pressures less than $14 \mathrm{~cm} \mathrm{H}_{2} \mathrm{O}$ as well as inspired $\mathrm{O}_{2}$ concentrations less than $30 \%$. PTV mode was not associated with an increased risk of hypocarbia in the regression model. Infants receiving minimal ventilation with mild lung disease on the first day of life were therefore more prone to hypocarbia.

We utilised data routinely collected by one centre within the multicentre trigger ventilation trial, which limited some of the analysis. Calculation of oxygenation indices was precluded as $\mathrm{PaO}_{2}$ data were not available. Oxygenation indices would have provided a better marker for severity of lung disease than $\mathrm{FiO}_{2}$ alone. Working within the design of the multicentre trigger ventilation trial provided two well matched groups with the exception of male gender. A larger proportion of male infants was randomised to the CMV group; however, the difference in proportions was not significant and male sex was not associated with hypocarbia in the regression model.

It is possible that the randomisation process selected infants with milder lung disease and consequently the large proportion of hypocarbic infants might be an overestimation of the problem. The two groups were well matched in terms of illness severity, both with median CRIB scores of 7, and the CRIB score did not feature as a significant variable in the regression model.

We considered whether inadvertent hypocarbia could have been an artefact of the ventilation protocol. Analysis of variance showed no significant difference between preand post-randomisation $\mathrm{PaCO}_{2}$ levels. Making the assumption that the pre-randomisation $\mathrm{PaCO}_{2}$ level reflects the attending clinician's chosen style of ventilation, we consider it unlikely that the guidance provided by the protocol was solely to blame for inadvertent hypocarbia.

Significant hypocarbia was defined as an arterial $\mathrm{PCO}_{2}$ level less than or equal to $3.33 \mathrm{kPa}$ $(25 \mathrm{~mm} \mathrm{Hg})$, based on the findings of two other studies. ${ }^{67}$ Calvert et al compared 15 preterm infants with periventricular leukomalacia (PVL) with 15 preterm controls without PVL and showed that infants with PVL had longer periods with $\mathrm{PaCO}_{2}$ readings below $3.33 \mathrm{kPa}$ during the first 72 hours of life. ${ }^{6}$ Wiswell et al showed that cumulative hypocarbia below a threshold level of $3.33 \mathrm{kPa}$ during the first day of life was the single factor independently related to the development of cystic PVL. ${ }^{7}$

Our infants on PTV received lower peak inspiratory pressures than infants on CMV during the first two days of life. The study of Hummler et al showed that infants on trigger ventilation required lower peak inspiratory pressures than those on intermittent mandatory ventilation to maintain a similar level of minute ventilation. ${ }^{13}$ The lower peak inspiratory pressures could be caused by a similar mechanism. Alternatively, this probably simply reflects the different weaning strategies, with lower peak inspiratory pressures before extubation in the PTV group.

The use of stepwise logistic regression identified those infants with the least requirements for ventilatory support as having the highest risk of hypocarbia, regardless of mode of ventilation. This suggests that a more aggressive approach to extubation and withdrawal of ventilation is required, particularly on day 1 . Our literature search provided no evidence of similar studies investigating the risk factors associated with hypocarbia in preterm infants.

The frequency of inadvertent hypocarbia on day 1 was of concern. We were unable to find comparable information from other units. Exposure to inadvertent hypocarbia in ventilated preterm infants on day 1 could be used as a measure of the effectiveness of ventilator management, as part of an audit of practice.

In conclusion, hypocarbia is an undesirable and frequently encountered consequence of ventilation. Preterm infants with mild hyaline membrane disease require careful monitoring 
of $\mathrm{PaCO}_{2}$ levels and timely weaning of ventilation to extubation to limit the risk of hypocarbia.

1 Leahy FAN, Cates D, MacCallum M, Rigatto H. Effect of $\mathrm{CO}_{2}$ and $100 \% \mathrm{O}_{2}$ on cerebral blood flow in preterm infants. F Appl Physiol 1980;48:468-72.

2 Wyatt JS, Edwards AD, Cope M, et al. Response of cerebral blood volume to changes in arterial carbon dioxide tension

in preterm and term infants. Pediatr Res 1991;29:553-7.

3 Greisen G, Munck H, Lou H. Severe hypocarbia in preterm infants and neurodevelopmental deficit. Acta Paediatr Scand 1987;76:401-4.

4 Graziani LJ, Spitzer AR, Mitchell DG, et al. Mechanical ventilation in preterm infants: neurosonographic and ventilation in preterm infants: neurosonographic
developmental studies. Pediatrics 1992;90:515-22.

5 Fujimoto S, Togari H, Yamaguchi N, et al. Hypocarbia and cystic periventricular leukomalacia in premature infants. Arch Dis Child Fetal Neonatal Ed 1994;71:F107-F110.

6 Calvert SA, Hoskins EM, Fong KW, Forsyth SC. Etiological factors associated with the development of periventricular leukomalacia. Acta Paediatr Scand 1987;76:254-9.

7 Wiswell TE, Graziani LJ, Kornhauser MS, et al. Effects of hypocarbia on the development of cystic periventricular leukomalacia in premature infants treated with highfrequency jet ventilation. Pediatrics 1996;98:918-24.

8 Ikonen RS, Janas MO, Koivikko MJ, et al. Hyperbilirubinemia, hypocarbia and periventricular leukomalacia in preterm infants: relationship to cerebral palsy. Acta Paediatr 1992;81:802-7.

9 Kraybill EN, Runyan DK, Bose CL, Khan JH. Risk factors for chronic lung disease in infants with birth weights of 751 to 1000 grams. F Pediatr 1989;115:115-20.

10 Garland JS, Buck RK, Allred EN, Leviton A. Hypocarbia before surfactant therapy appears to increase bronchopulmonary dysplasia risk in infants with respiratory distress syndrome. Arch Pediatr Adolesc Med 1995;149:617-22.

11 Mehta A, Callan K, Wright BM, Stacey TE. Patienttriggered ventilation in the newborn. Lancet 1986;2:17-19.

12 Greenough A, Hird MF, Chan V. Airway pressure triggered ventilation for preterm neonates. F Perinat Med 1991;19:471-6.

13 Hummler H, Gerhardt T, Gonzalez A, et al. Influence of different methods of synchronised mechanical ventilation on ventilation, gas exchange, patient effort, and blood
pressure fluctuations in premature neonates. Pediatr Pulmonol 1996;22:305-13.

14 Greenough A, Greenall F. Patient triggered ventilation in premature neonates. Arch Dis Child 1988;63:77-8.

15 Baumer JH. International randomised controlled trial of patient triggered ventilation in neonatal respiratory distress syndrome. Arch Dis Child Fetal Neonatal Ed 2000;82:F5-F10.

16 The International Neonatal Network. The CRIB (clinical risk index for babies) score: a tool for assessing initial neonatal risk and comparing performance of neonatal intensive care units. Lancet 1993;342:193-8. 